\title{
Editorial
}

\section{Infection Surveillance and Control and Coronary Artery Bypass Surgery}

\author{
James T. Lee, MD, PhD
}

Several years ago, I joined colleagues from surgery, infection control, and epidemiology at Jackson Hole, Wyoming, for a 3-day boondoggle. We were hosted by a multinational corporation. Fly-fishing, horseback riding, hiking, and shooting rapids on the Snake River were interspersed with multiple sessions of "visioning," "thinktanking," and "sharing" regarding the future of surgical infection control. In these activities we were joined by corporate scientists and marketing mavens. The corporation had retained an unctuous "facilitator" to run the show, and, at the compulsory opening night welcome party held in a rustic lodge, she asked that each doctor stand before the group to recite his or her credentials. Ice tinkled in cocktail glasses, and a fire roared in the giant hearth as we took our turns. A friend of mine, who happens to be one of the best cardiac surgeons in the United States, brought down the house. Raucous laughter followed his comment: "I don't know why you invited me and my wife, but it looks like we're gonna have a good time. Let me just say I'm a cardiac surgeon, and I'm opposed to infections of all kinds. Thank you very much."

Postoperative infections of all kinds can ruin recovery from the most meticulously performed coronary artery bypass operations. In recent years, there has been growing support for organized infection surveillance of cardiacsurgery patients, coupled with thoughtful processimprovement initiatives as indicated.

A recent article in this journal will be cited in the future as evidence that surgical-site infection (SSI) surveillance and control programs can add tangible value in cardiac-surgery practices. McConkey and coworkers at Barnes Hospital in St Louis, Missouri, tried to determine whether a newly activated surveillance and control program favorably influenced SSI risk. ${ }^{1}$ A clever strategy was used and, had the paper been a Lassie movie, regression analysis would certainly have played the starring role of the heroic dog who saves the day. Given the essential impossibility of testing mixed infection-prevention methods in a controlled trial (including the effect of having a surveillance function), the authors used regression models to allow disqualification of factors that might be adduced by skeptics like me to explain away any beneficial secular impact (ie, decreased infection rates) of surveillance and control activity. The article is not perfect, but it is important and its details are rich. It is not just another recital of one hospital's postoperative infections, and surveillance enthusiasts should dissect it critically in a quiet room. A sharp number 2 pencil and note pad should be kept handy. Both will be needed.

Surveillance was performed after 2,230 coronary artery bypass operations during a baseline year and 3 consecutive follow-up years ending with 1994. Annual infection rates were not merely compared to see what happened after numerous prevention modalities were structured and activated. Instead, the rates were "cleaned up" using several maneuvers that come from the analytical epidemiology toolbox.

Final adjusted risk models showed that there had been no significant improvement relative to the index year for any of three annual rates for "deep chest infections," an unorthodox term that included mediastinitis, the much feared and most expensive nosocomial infection in modern cardiac bypass surgery. The significant improvement discovered by these authors, after removing the influences of several confounding variables, was a striking decrease in the annual incidence rate for leg-vein harvest-site infections. This represents genuine improvement. It can be argued that the improvement touched all four healthcare outcome components (clinical, economic, patient satisfaction, and patient functional status). Readers should be 
extremely curious to know if the improvement was sustained in the period from 1995 to 1998 .

It would have been helpful to know whether the Barnes surgeons did things differently in the study years with regard to their closures of leg incisions. Such surgical wounds are sometimes complicated by delayed healing that may be partly technique-related. Did surgeons use skin staples, percutaneous sutures, or subcuticular closures for their leg incisions? Were subcutaneous sutures used uniformly and, if so, what suture material was used? These and related details could have been easily logged prospectively, but apparently they were not. It would obviously be a daunting project now to dig out these particulars retrospectively. Who wants to go back and read through, line by line, several thousand operative dictations?

After saphenous vein harvesting, one antecedent of both postoperative infection in the leg wound and its falsepositive diagnosis can be mechanical healing failure in the skin-skin interface. Such flaws are often occult until after inpatient discharge, usually involve isolated and short incision segments (perhaps $1-3 \mathrm{~cm}$ ), and are not rare in older patients who have peripheral vascular disease or diabetes mellitus. Challenged perfusion provably does not hasten healing of a primarily closed skin incision, peri-incisional edema does not help matters, ischemic necrosis of apposed skin edges does occur in this setting, and what a layperson might call "open sores" may develop at one or more points in the long axis of a closed incision. Are such clinical outcome flaws infections, or should they be classified separately as wound-healing failures? Even experienced infection control nurses know that diagnosing saphenous vein harvest-site infections can be highly subjective: not every infected leg incision exudes pus, not every ugly leg incision is infected, and not every scab has evil portent. McConkey et al had no choice but to presume that inaccuracies of vein harvest-site infection diagnosis were present at a uniform level in every study year. This critical premise hovers uncomfortably in the background of their research contribution, because the leg-incision-infection category was the only domain of significant improvement.

I am uneasy that no formal plan existed for the identification of postdischarge infections during a defined window (eg, 30 days). When there is no set protocol, it is not impertinent to suspect fluctuating detection efficiency in sequential time periods, a germane issue no matter whose medical center produces the data. Approximately $70 \%$ of the patients were referred from outside the St Louis area, suggesting that many patients had some follow-up examinations in the offices of distant cardiologists. Do we know if those nonsurgeons zealously sought findings of infection with uniform intensity over the study years? As I understand it, the detection modus operandi included using a computer to spot evidence that positive cultures had been obtained by caregivers. A hidden premise is that such professionals exercised the option to send a culture at a uniform rate in every study year. How do we know this premise is sound? How many infected leg incisions were registered after finding positive cultures of granulation tis- sue in dehiscent but actually noninfected skin closures? Could decreased leg incision infection rates over 3 years be partly explained by decreasing false-positive diagnosis rates in each study year as surveillance personnel honed their skills? Readers will note the authors' explicit statement that National Nosocomial Infection Surveillance definitions for diagnosis were used, yet a report of culture growth is not usually needed to diagnose infection at a surgical site. I do not think that using a computer network to spot cultures necessarily imposed a year-wise differential accuracy that fortuitously explains in one fell swoop both the rate improvements for leg-incision infections and the lack of rate improvements for deep chest infections.

Some aspects of the paper seem misleading. In the abstract, the unmistakable tone is that multiple things got better over the study period, but not everything got better, as readers discover later in Table 3 . Then comes Table 4, a dramatic punch line for the entire story, where only a few adjusted odds ratio contrasts were significantly decreased when four kinds of outcome for each of 3 study years were contrasted to 1991 index values. Even without using my new Mont Blanc half-glasses, I saw lots of odds ratio confidence intervals that covered both sides of 1.0 (perhaps I misinterpreted the table).

The authors unwittingly injected confusion in three ways: (1) by using a peculiar terminology that unfortunately resembles Centers for Disease Control and Prevention (CDC) nomenclature, (2) by using CDC terminology incorrectly in some places but correctly in others, and (3) by allowing internal inconsistency in use of their own terminology. To illustrate just one example of the latter, the "Results" section of the abstract contains a sentence in which deep chest infections are differentiated from mediastinal infections; yet, in the body of the article, we read that deep chest infections refer to combined counts of the deep incisional infections at the sternum and organ/space infections in the chest. Mediastinitis is one kind of organ/space SSI, so the bewildering contradiction should have been caught by manuscript reviewers. Careful readers will detect some other examples of contradictions and fuzzy meaning that impart a Sunday New York Times crossword puzzle theme to the work of reading the article. Curiously, "superficial incisional SSI," a formal CDC term, never appears as a data category, although both leg incisions and pre-sternal soft-tissue incisions commonly are affected by that complication, begging two questions: How many superficial incisional SSI occurred each year at the chest incision? How many leg incision infections at Barnes Hospital were deep incisional SSI (ie, invasion of fascia or muscle in the wound bed)? Based on my experience in one of the largest surgicalwound infection surveillance registries in the country, my guess for the second answer is "very few." In Table 2, which nicely summarizes relative risks for a wide range of univariate comparisons, it appears that the authors drew a distinction between SSI and deep chest infection.

I wish that a five-column, four-row data table had been added, listing the numbers of infections logged each year according to "all SSI," "deep incisional SSI (leg)," 
"superficial incisional SSI (leg)," "superficial incisional SSI (sternal)," and "composite chest" categories. In a footnote to such a user-friendly table, the authors could have pled the case for creating their own composite category that merged all surgical chest infections except superficial incisional infections, a decision forced by practical considerations that are unique to cardiac operations, and a ploy with which I have absolutely no disagreement.

Confusing terminology issues not only hamper facile comprehension of a paper but also can irretrievably fog up the accurate generalization of its most important findings. I fear that the authors fell into a linguistic trap (also visited frequently by surgical colleagues) in using incorrectly the terms deep site and superficial site throughout a publication peppered with more than 30 uses of the abbreviation SSI. What can be stated with certainty here?

Surgical-site infection is a term coined by the CDC in 1992. A possibly widespread misunderstanding (and not just by surgeons) seems to persist regarding the meaning of the term surgical site. The crux of the problem here is that site has a both general meaning (location, place, etc) and a particularized meaning in CDC terminology. In the formal CDC nomenclature scheme, ${ }^{2}$ two kinds of sites are explicitly defined: incisional and organ/space. The terms deep and superficial were additionally defined to designate two anatomic levels of incisional SSI. The two terms do not refer to two kinds of sites! The meaning of organ/space site is also defined exactly in the CDC scheme. The modifiers deep and superficial have no connection with organ/space SSIs. Thus the terms superficial site and deep site are not only meaningless but also are especially confusing because they sound like CDC labels.

The preceding comments are going to be regarded by some readers of this editorial as pedantic carping. However, the "Conclusions" section of the McConkey article's abstract, which will be read by plenty of people online after snagging the paper's citation in a MEDLINE search, states the following: "We observed significant reductions in SSI rates of deep and superficial sites in CABG surgery following implementation of a comprehensive infection control program."

What did that sentence mean? After numerous readings, I could not find anything in the article that substantiates significant improvement in rates for anything deep, either deep incisional SSI (as in the formal CDC term) or deep chest infection (as in the authors' neologism). The authors' unadjusted rate trend tests showed no significant rate decrease for their deep chest infections. They also compared adjusted odds ratio values for years 2,3 , and 4 to index values of 1.0 for 1991, finding significance only for "leg infections" (for all 3 years) and "any SSI" (only for 1994) in those contrasts. It seems a fair deduction that their conclusion ("... significant reductions in SSI rates of deep and superficial sites ...") goes beyond their data, even if we presume that superficial sites meant superficial incisional SSI and that deep sites meant deep chest infections plus (unreported) deep incisional SSI for legs.
I am reluctant to think that the authors were attempting a grammatical parlor trick, but I do think they got ensnared in their own terminology conflicts. Ironically, one of the benefits of reading the McConkey article (and hopefully this editorial) may be that authors, reviewers, and editors are sensitized to the pressing need for exact use of SSI terminology conventions in consistent fashion. Postoperative infection epidemiology is intrinsically complex already, and the encryption of first-class research findings must be avoided.

It will puzzle some readers that death was made a response variable in one of the regression models used to study surgical-infection epidemiology. Apparently, 30-day postoperative mortality was meant by "death," but even had that orthodox definition been stated, inclusion of death rates seems a non sequitur, unless the finding of insignificant mortality change for each study year relative to 1991 was revealed to anticipate and disarm a cynical inference that a whole bunch of easy cases must have progressively populated the Barnes case loads from 1992 to 1994 . Of course, multiple ways to die after a cardiac operation have no relation to the presence or absence of nosocomial infection.

Finally, I was chagrined to find a misinterpretation of my hospital's use of surveillance data. We have not reported surgeon-specific infection rates (as alleged by the authors) in the entire 22-year history at Minneapolis. The ultimate personnel stratification for our surgical wound infection rates has consistently been by surgical specialty. 3,4

A dozen other nits might be picked, but I still regard the author's work as seminal and ambitious. They put on the full-court press and showed that, by correcting for the effects of some plausible risk-influencing variables, "practice year" can be usefully viewed as an independent variable, with annual infection rate a response variable. Obviously, practice year is a surrogate for possibly numerous concealed variables; the authors had to assume that infection risk heterogeneity within that label was constant or at least had no net effect of decreasing the risk over time-maybe the most important untestable assumptions in a chain of reasoning that led to this tentative conclusion: setting up a comprehensive surveillance and infection control program at Barnes Hospital favorably influenced one aspect of care quality in coronary artery bypass surgery.

\section{REFERENCES}

1. McConkey SJ, L'Ecuyer PB, Murphy DM, Lee TL, Sundt TM, Fraser VJ. Results of a comprehensive infection control program for reducing surgical-site infections in coronary artery bypass surgery. Infect Control Hosp Epidemiol 1999;20:533-538.

2. Horan TC, Gaynes RP, Martone WJ, Jarvis WR, Emori TG. CDC definitions of nosocomial surgical site infections, 1992: a modification of CDC definitions of surgical wound infections. Infect Control Hosp Epidemiol 1992;13:606-608.

3. Olson MM, Lee JT Jr. Continuous 10-year wound infection surveillance. Results, advantages, and unanswered questions. Arch Surg 1990;125: 794-803.

4. Lee JT, Olson MM. Wound infection surveillance for 85,260 consecutive operations. Journal of Surgical Outcomes 1999;2:27-42. 